\title{
Three-dimensional sloshing in a scaled membrane LNG tank under combined roll and pitch excitations
}

\author{
Min Luo ${ }^{\mathrm{a}}$, Xin Wang ${ }^{\mathrm{a}}$, Xin Jin ${ }^{\mathrm{b}}$, Bin Yan ${ }^{\mathrm{c}, *}$ \\ ${ }^{a}$ Zienkiewicz Centre for Computational Engineering, College of Engineering, Swansea University, Swansea \\ SA1 8EN, United Kingdom \\ ${ }^{\mathrm{b}}$ College of Energy, Chengdu University of Technology, Chengdu, Sichuan 610059, China \\ c Department of Civil and Environmental Engineering, National University of Singapore, Kent Ridge, Singapore \\ 117576, Singapore
}

\begin{abstract}
This paper experimentally investigates the three-dimensional sloshing in a membrane-type LNG (liquefied natural gas) tank under combined roll and pitch excitations. Seven groups of roll and pitch amplitudes are studied. For each group, the roll and pitch have the same frequency, and around ten frequencies are tested in a frequency band that ranges from 0.5 times of the resonance frequency in the length direction of the tank to 1.4 times of the resonance frequency in the tank breadth. The characteristics of the sloshing waves and impact pressures are analysed in detail. It is found that the steady-state sloshing waves can be classified into four patterns: the length-dominant wave, swirling wave, diagonal wave, and breadth-dominant wave. Highlighted is the swirling wave that is observed in most of the cases where the roll and pitch amplitudes are significant and the excitation frequency locates between the resonant frequencies in the two directions. It is hypothesized that the rotational motion of the tank imposes necessary initial conditions to trigger the swirling wave. The swirling wave is always associated with wave impingements at tank corners and induce violent impact pressures. The practical implementation is that reinforcements of the membrane layer should be added or the sloshing wave should be suppressed near the tank corner to mitigate the damages to the interior layer of the membranetype LNG tank.
\end{abstract}

Keywords: 3D sloshing; membrane LNG tank; rotational excitation; swirling wave; wave pattern

\section{Introduction}

The global increasing needs are continuously driving the demand for natural gas. The liquefied natural gas (LNG) is easier to store and transport as compared to the gaseous form and hence is widely gaining popularity in the energy industry. Among the various LNG carriers, the membrane type in the octagonal prismatic shape has been increasingly used because of the relatively low cost in construction

\footnotetext{
* Corresponding author.

E-mail: yanbin963934493@163.com
} 
and the high volume utilization (Mokhatab et al., 2013). One major design factor for the membrane tank is the LNG sloshing wave and the associated impact loads. The high impact pressure may cause serious damages to the interior layer of the tank (Wang, 2010) and the sloshing force may generate large overturning moments that adversely affect the navigation stability of the ship (Luo et al., 2016).

The characteristics of sloshing waves and sloshing-induced loads are closely related to the filling level. In general, the sloshing waves in low and intermediate filling levels can be more violent and induce larger impact pressures (DNV, 2016). To avoid violent impact loads, therefore, the containment systems of some LNG carriers have been designed with filling level restrictions, which is also termed the barred filling level (DNV, 2016; Lloyd'sRegister, 2008). The filling level restriction, however, constraints the operation and trading flexibility especially for the LNG-FPSO (floating production storage and offloading) unit, in which the change of filling level during the exploration and offloading operations cannot be avoided (Malenica et al., 2017; Zhao et al., 2018). This necessitates the studies of violent sloshing in the intermediate filling levels.

The middle-filling-level sloshing in different tanks under different excitations has been the topic of numerous researches. A large portion of these studies focused on the sloshing in rectangular tanks under single-degree-of-freedom (longitudinal) translational (Koh et al., 2012; Liu and Zhang, 2019; Liu et al., 2016; Lu et al., 2018; Meng et al., 2020; Pal and Bhattacharyya, 2010; Xue et al., 2017; Xue and Lin, 2011; Zheng et al., 2017) and rotational (Bulian et al., 2014; Chen et al., 2013; Delorme et al., 2009; Souto-Iglesias et al., 2015) excitations. In these cases, the simplification of two-dimensional (2D) waves gives satisfactory predictions of the sloshing wave profile and pressure. In reality, LNG containers are subjected to multi-degree-of-freedom excitations and suffer from three-dimensional (3D) sloshing. 3D sloshing is much more complex and imposes more intensive destructive power than 2D sloshing (Zhao et al., 2018). Substantial researches have been carried out to improve the understanding of 3D sloshing. Similar to the 2D work, the majority of 3D studies investigated the sloshing phenomena in rectangular tanks (Bai et al., 2015; Faltinsen et al., 2005; Jin and Lin, 2019; Liu and Lin, 2008; Wu et al., 2013; Zhao et al., 2018). For the membrane-type LNG tank, the chamfers affect the sloshing wave motion and reduce the impact load by changing the impact angle (DNV, 2016). To reveal the sloshing characteristics in membrane LNG containers, therefore, it is necessary to study the 3D sloshing in prismatic tanks.

Among the various parameters of sloshing, the sloshing wave profile is fundamental in analysing the sloshing dynamics and has been investigated extensively. For example, the sloshing wave profile in a square tank induced by longitudinal and diagonal horizontal motions was studied analytically in Faltinsen et al. (2005). The steady-state sloshing waves were classified into four types, i.e. the planar, diagonal, chaotic, and swirling waves. Similar sloshing scenarios were studied numerically in Wu and Chen (2009), which classified the sloshing waves into five types, i.e. the diagonal, single-directional, square-like, swirling, and irregular waves. This classification divided the planar wave in Faltinsen et al. 
(2005) into the single-directional and square-like waves. This study was extended to introduce the heave excitation in Chen and $\mathrm{Wu}$ (2011). It was found that the heave excitation has significant influences on the sloshing wave pattern. A subsequent study in Wu et al. (2013) examined the generation of swirling wave and suggested that the swirling wave could only be generated for a partially filled tank excited at the near-resonant frequency with oblique horizontal excitations.

For the prismatic tank, Arai et al. (2018) studied the sloshing in membrane LNG tanks under unidirectional sway excitations. It is found that the swirling waves could be excited in middle to low filling levels when the length-to-breadth ratio of the tank is close to one. The work of Luo et al. (2016) investigated the sloshing in a membrane tank, whose length-to-breadth ratio is around 1.4, under oblique horizontal excitations of heading angle $45^{\circ}$. The excitation frequencies $f_{\mathrm{L} 0}$ and $f_{\mathrm{B} 0}$ (the fundamental natural frequencies of the sloshing system in the length and breadth directions) and the third one between these two were tested, generating the length-dominant, breadth-dominant and diagonaldominant waves, respectively. However, three frequencies are not sufficient to reflect the variation of sloshing wave patterns over the entire frequency band. This issue was addressed at the preliminary stage of the current study. Based on the same experimental setup in Luo et al. (2016), more excitation frequencies (a wider frequency range and denser frequency values) and two more heading angles of the excitation (i.e. $16.0^{\circ}$ and $71.5^{\circ}$ ) are tested, resulting in 30 cases in total. The patterns of the steady-state sloshing waves are summarized in Fig. 1. All the studied case only show the same three wave patterns presented in Luo et al. (2016), and the swirling wave is not observed. As discussed above, real LNG carriers are subjected to rotational motions. Are the wave patterns induced by rotational excitations the same as those excited by translational motions? This question is still not resolved yet and will be addressed in the present study.

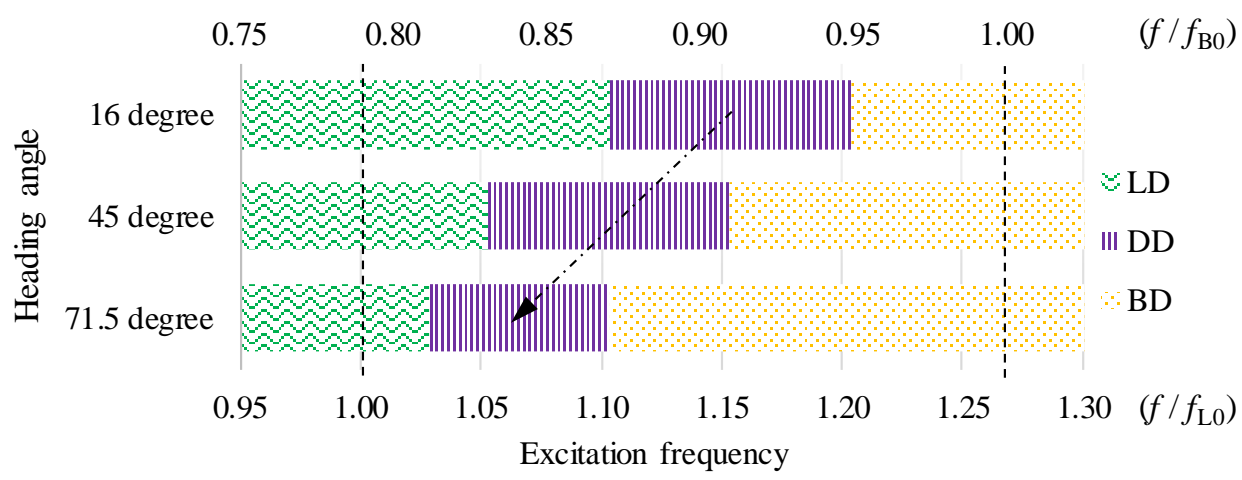

Fig. 1. Variation of sloshing wave pattern with excitation frequency and heading angle under oblique translational excitation (LD: length-dominant; DD: diagonal-dominant; BD: breadth-dominant)

With the engineering and research background illustrated above, this work aims to investigate the violent 3D sloshing in a scaled membrane LNG tank under combined roll and pitch excitations, which has been rarely reported in literatures. Specifically, this study has two objectives: (1) to classify the wave patterns under rotational excitations of different amplitudes and frequencies; (2) to examine the 
characteristics of sloshing pressure in different wave patterns. The sloshing wave pattern that applies the most violent impact pressures is highlighted. This work is an important supplement to previous studies that mainly considered the rectangular tank and/or translational excitations. The experimental approach is used because this is considered to be the most reliable approach in predicting violent sloshing and the associated impact pressures (DNV, 2016). In the following, the experimental methodology will be introduced in Section 2; the results and discussion are elaborated in Section 3; concluding remarks are made in Section 4.

\section{Experimental methodology}

\subsection{Experimental setup}

The experiments were conducted on a rotational simulator that can apply two-degree-of-freedom rotational motions as shown in Fig. 2. This setup was successfully used to study the water sloshing under resonant and random excitations with the experimental data utilized to validate a numerical model (Luo and Koh, 2017; Luo et al., 2016). The current study expands the range of excitation amplitude and frequency and aims to examine the sloshing mechanism under a broad range of excitation conditions. For completeness of illustration, the experimental setup is recapped here. As sketched in Fig. 3, the rotations around $x$ and $y$ axes are defined as pitch and roll, respectively. Driven by two alternatingcurrent motors, the simulator can apply regular/irregular pitch-only, roll-only and coupled pitch and roll motions with Point $o$ (at a certain distance below the centre of the simulator platform) as the pivot. The maximum pitch/roll excursion is $\pm 25^{\circ}$ and the maximum angular velocity $25^{\circ} /$ second. The excitations considered in this study are all within the specification range. Under coupled pitch and roll motions, the resultant motion of the simulator should be a rotation around an axis in the $x-o-y$ plane, and the orientation of the axis is dependent on the amplitudes and phase angles of the pitch and roll components. In this study, a phase angle of $180^{\circ}$ was applied to both roll and pitch. Hence the actual rotation axis is in the third quadrant of the $x-o-y$ plane as indicated by the shadowed area in Fig. 3a and b. Note that the rotation centre of the platform is outside of the tank. This mimics the LNG tanks at both sides of an LNG ship because when a ship undergoes rotational motions, the rotational centre of the ship is outside the side tanks. More details of the simulator can be found in Luo et al. (2016).

The sloshing tank used in this study is a 1:80 scale model of a real membrane-type LNG tank, and its dimensions are shown in Fig. 4. The tank was made of Plexiglas of thickness $10 \mathrm{~mm}$. It was ensured that the deformation of tank walls under sloshing impacts, i.e. the hydroelastic effect, is negligible. The tank was fixed to the simulator platform with the centre of the tank bottom coinciding with the centre of the platform (see Fig. 2 and Fig. 3a). For ease of discussion, the left side wall on which pressure sensors were installed is called "sidewall", and the front wall that is close to the pressure sensor installed at the corner is called "front wall". Under excitations of different amplitudes/frequencies, the sloshing waves show different dominant propagation routes, i.e. moving along the length, breadth and diagonal 
of the tank and swirling around the side walls of the tank. These wave patterns are sketched in Fig. 3c and will be elaborated in Section 3.2.

A video camera was placed in front of the tank (see Fig. 2) to record the sloshing wave motions. To enhance the clarity of the video image, colour food dye was added to water. Snapshots taken from the video provide the experimental wave profiles. The impact pressure induced by sloshing waves is among the most important factors in engineering design and is investigated in the present study. It was reported in Lloyd'sRegister (2008) that the upper knuckle of the lower hopper of a side wall suffers from serious sloshing impacts. Therefore, the sloshing pressures in this area are examined. Specifically, the sloshing pressures at the middle of the sidewall ( $\mathrm{P}_{1}$ in Fig. 4$)$ and near the corner $\left(\mathrm{P}_{2}\right)$ were measured. The WIKA S-10 gauge pressure sensors were used. The pressure range of the sensor is $100 \mathrm{mbar}\left(10^{4} \mathrm{~Pa}\right)$, accuracy $0.25 \%$ full scale and response time smaller than 1 millisecond (a sampling frequency of $2 \mathrm{kHz}$ was used in the experiments). The diameter of the circular measurement diaphragm of the sensor is $3.5 \mathrm{~mm}$. These specifications enable the sensors to measure localized transient (rapidly varying) peak pressures. The sensor is based on a piezoresistive measuring element and hence can capture the pressure applied on it, irrespective of whether the pressure change is caused by water, air or water-air mixture. This ensures the applicability of the pressure sensors to air entrapment/entrainment that always happens in violent sloshing scenarios. All the pressure signals were recorded by an oscilloscope. Note that this way of installing the two sensors enables the capturing of the pressure characteristics of different sloshing patterns, and hence provides sufficient data for the second research objective of this study.

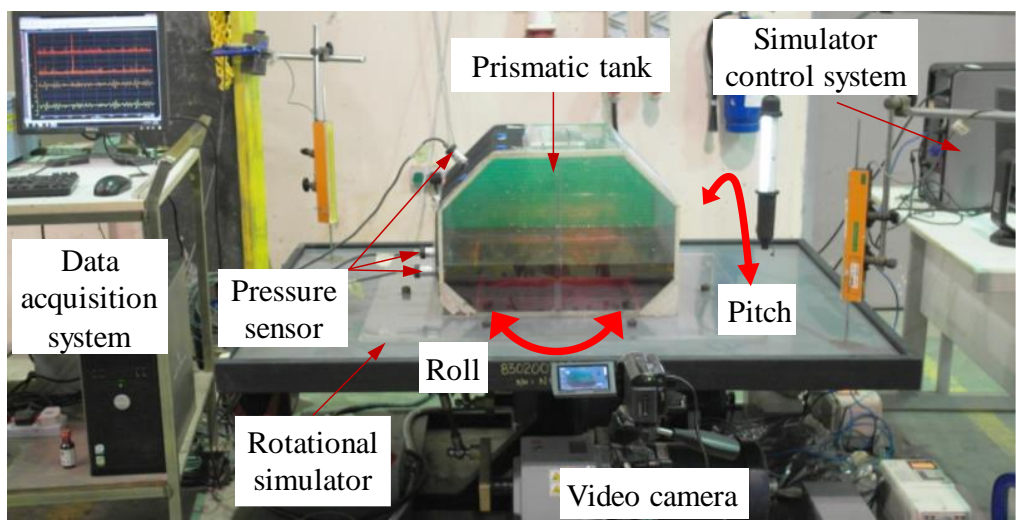

Fig. 2. Setup of 3D sloshing under the coupled roll and pitch excitations (the figure is based on those in Luo et al. (2016) and Luo and Koh (2017)).

To test the reliability of the experimental setup, a repeatability analysis was conducted based on the sloshing in 50\% filling level under the roll-only resonant excitation. This case involved violent sloshing waves. In the four repeats, the peaks of sloshing pressure after the eighth cycle show some variations (the maximum difference is less than 15\%). This is reasonable for the violent sloshing case (Bulian et al., 2014; Souto-Iglesias et al., 2012). For the statistically averaged peak pressure (see Section 3.3 for details) that matters in this study, its value at $\mathrm{P}_{2}$ (the more critical case with more uncertainties) is 1741.9 

$\mathrm{Pa}$, 1714.6 $\mathrm{Pa}, 1733.3 \mathrm{~Pa}$ and 1744.4 $\mathrm{Pa}$, respectively, in the four repeats. The mean, standard deviation and coefficient of variation are $1733.5 \mathrm{~Pa}, 11.7 \mathrm{~Pa}$ and $0.7 \%$, respectively. This shows the good repeatability of the experiments and hence the reliability of the experimental setup.

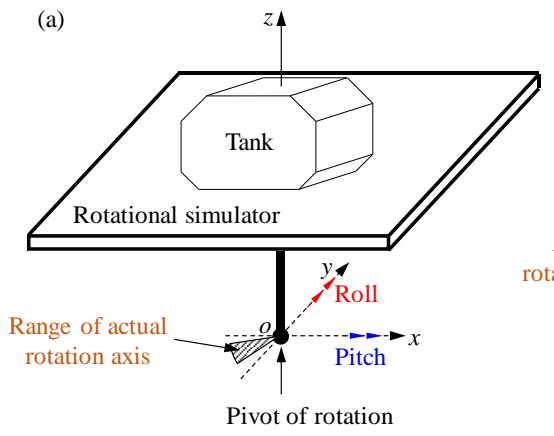

(b)

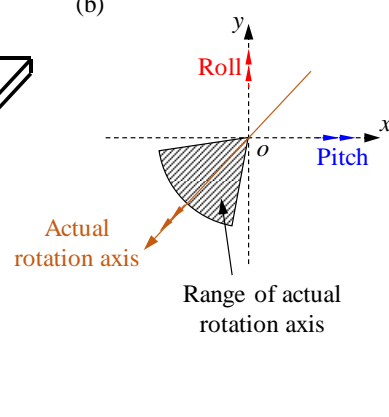

(c) Swirling wave

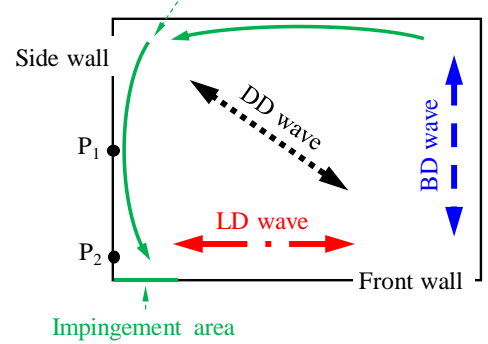

Fig. 3. Schematic view of the experimental setup: (a) perspective view of the whole setup; (b) the convention for roll and pitch in a plain view; (c) routes of different sloshing waves in a plain view

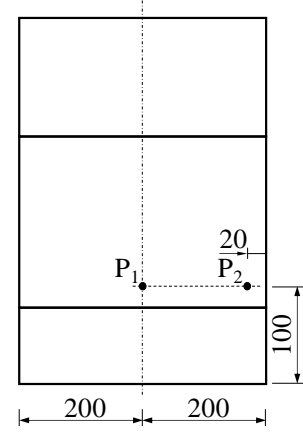

(a) Left view

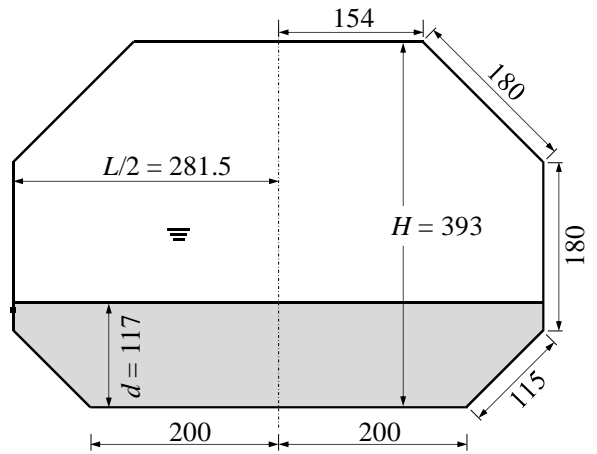

(b) Front view

Fig. 4. Dimensions of the laboratory-scale membrane-type LNG tank

\subsection{Experimental parameters}

The introductory section has explained the necessity to study the sloshing impact at the intermediate filling level. Preliminary studies of the current work showed that the filling level of $30 \% \mathrm{H} H$ is the tank height) produces the highest impact pressure near the upper knuckle of the lower hopper. Therefore, this water level was selected in this study. The fundamental natural frequencies of the sloshing system in the length and breadth directions can be estimated using the linear wave theory (Faitinsen, 1978). They are $0.891 \mathrm{~Hz}$ and $1.189 \mathrm{~Hz}$, respectively, denoted by $f_{\mathrm{L} 0}$ and $f_{\mathrm{B} 0}$. Note that the actual natural frequencies can deviate from the estimated ones due to the irregular shape of the tank and the significant nonlinearity of the violent sloshing. In this study, the harmonic (regular) roll and pitch motions were utilized, and the roll and pitch were of the same frequency in each case. The excitation frequency (denoted as $f$ ) varied from $0.5 f_{\mathrm{L} 0}$ to $1.4 f_{\mathrm{B} 0}$ with more refined test frequencies conducted near the transition from one wave pattern to another.

The combination of roll and pitch amplitudes is another main parameter of study. Seven excitation groups (EGs) were investigated, as shown in Table 1. The ratio of roll amplitude to pitch amplitude 
decreases from EG\#0 to \#6. Since the roll and pitch were of the same frequency and have the same phase angle (180 degrees), the resultant motion of the platform is the rotation around an axis in the third quadrant of the $x-o-y$ coordinate system as shown in Fig. 3. This is somewhat analogical to the heading angle of an LNG ship in the sea. Specifically, the pitch-only excitation corresponds to the head sea, the roll-only excitation is for the beam sea, and a combination of these two resembles the bow sea.

Table 1 Roll and pitch angles in seven excitation groups

\begin{tabular}{ccccccccc}
\hline EG\# & 0 & 1 & 2 & 3 & 4 & 5 & 6 \\
\hline $\begin{array}{c}\text { Rotation } \\
\text { amplitude } \\
\begin{array}{c}\text { (degree) } \\
$\cline { 2 - 9 }\end{array}\end{array} & Roll & 2 & 2 & 2 & 2 & 2 & 1.5 & 1 \\
\cline { 3 - 9 } & Pitch & 0 & 0.5 & 1 & 1.5 & 2 & 2 & 2 \\
\hline
\end{tabular}

68 cases with different rotation amplitudes and excitation frequencies were studied. Each case was tested for $36-60$ cycles depending on the features of sloshing waves and pressure signals. The water was selected as the sloshing media as its sloshing characteristics are very similar to those of LNG (Lee et al., 2007). This brings conveniences in conducting the experiments.

\section{Results and discussion}

\subsection{Transient and steady-state phases}

The ensemble-domain analysis of sloshing pressure in Bulian et al. (2014) showed that an initial transient stage exists in terms of the ensemble distribution of peak pressures and after that, the peak pressures tend to achieve a steady state from the probabilistic point of view. The 3D sloshing in this study also shows the transient and steady-state phases. They are identified by analysing the sloshing waves and pressure time series, the former providing a qualitative assessment while the latter enabling a quantitative evaluation. In the analysis of pressure, the pressure peaks in a time series are extracted and labelled as $p_{\mathrm{p} 1}, p_{\mathrm{p} 2} \ldots p_{\mathrm{p} k} \ldots p_{\mathrm{p} N}$. For the $k$-th peak, the average value of the data points from $p_{\mathrm{p} k}$ to $p_{\mathrm{p} N}$ is evaluated and labelled as $p_{\mathrm{m} k}$. If the relative difference between $p_{\mathrm{p} k}$ and $p_{\mathrm{m} k}$ is less than a threshold value (5\% is selected), the $k$-th cycle is the starting cycle of the steady state.

For illustration, the pressure histories of the case $f=1.055 f_{\mathrm{L} 0}\left(0.94 \mathrm{~Hz}, 0.791 f_{\mathrm{B} 0}\right)$ are presented in Fig. 5. The amplitudes of both $p_{1}$ and $p_{2}$ increase in the first seven cycles (transient phase) and approach to the values comparable to those in the subsequent cycles (steady-state phase). In some other cases, however, the sloshing waves in the transient phase are more violent than those in the steady state. This is exemplified by Fig. 6, which shows the pressure histories of the case $f=1.145 f_{\mathrm{L} 0}(1.020 \mathrm{~Hz}, 0.858$ $f_{\mathrm{B} 0}$ ). The major reason for the violent sloshing in the transient phase is the swirling wave, which will be detailed in the following sections. In this study, the transient phase is found to last for $5-9$ cycles, being consistent with the observation in Bulian et al. (2014). The wave pattern and pressure of the sloshing waves in the transient and steady-state phases will be elaborated. 


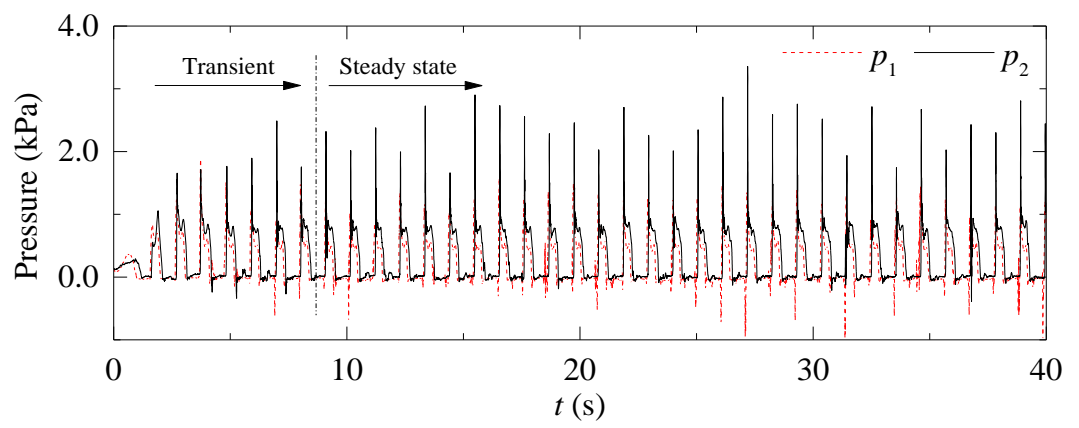

Fig. 5. Time histories of $p_{1}$ and $p_{2}$ for the case $f=1.055 f_{\mathrm{L} 0}\left(0.94 \mathrm{~Hz}, 0.791 f_{\mathrm{B} 0}\right)$ in EG\#3

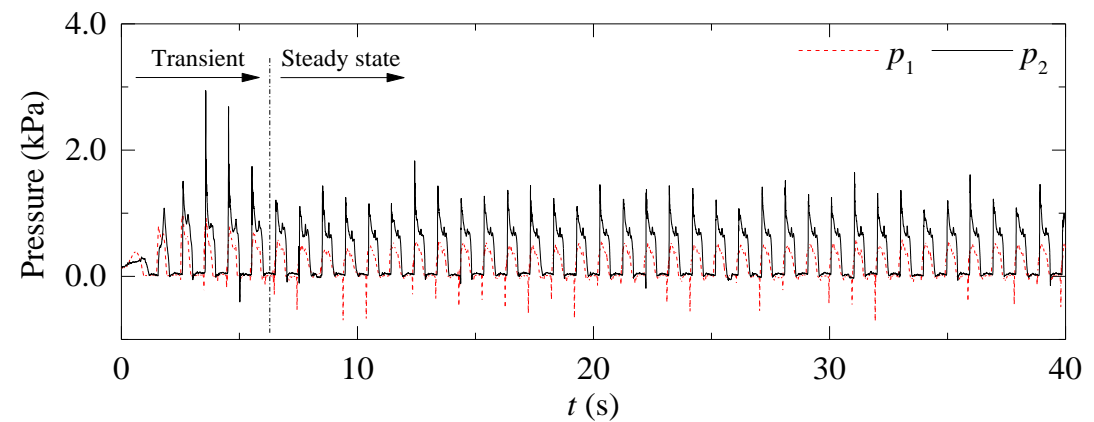

Fig. 6. Time histories of $p_{1}$ and $p_{2}$ for the case $f=1.145 f_{\mathrm{L} 0}\left(1.020 \mathrm{~Hz}, 0.858 f_{\mathrm{B} 0}\right)$ in EG\#3

3.2. Sloshing wave pattern

The sloshing wave pattern is analysed by scrutinizing experimental videos and analysing sloshing pressures. It is found that the steady-state sloshing waves of a case behave a certain pattern, while the waves in the transient phase are more complex and involve the transition from one pattern to another. In the following, the patterns of the steady-state sloshing waves are classified and the key features of the transient sloshing waves are discussed.

\subsubsection{Wave patterns in the steady-state phase}

The pattern variation of the steady-state sloshing waves with excitation frequency in all seven excitation groups is presented in Fig. 7. In general, the steady-state waves show four distinct patterns (see the sketches in Fig. 3c): the length-dominant (LD) wave that moves in the length direction of the tank; the swirling-dominant (SD) wave that travels in a swirling route in the tank; the diagonal-dominant (DD) wave that moves along one diagonal of the tank; the breadth-dominant (BD) wave that travels in the breadth direction of the tank. From Fig. 7, a series of observations can be made as follows: 


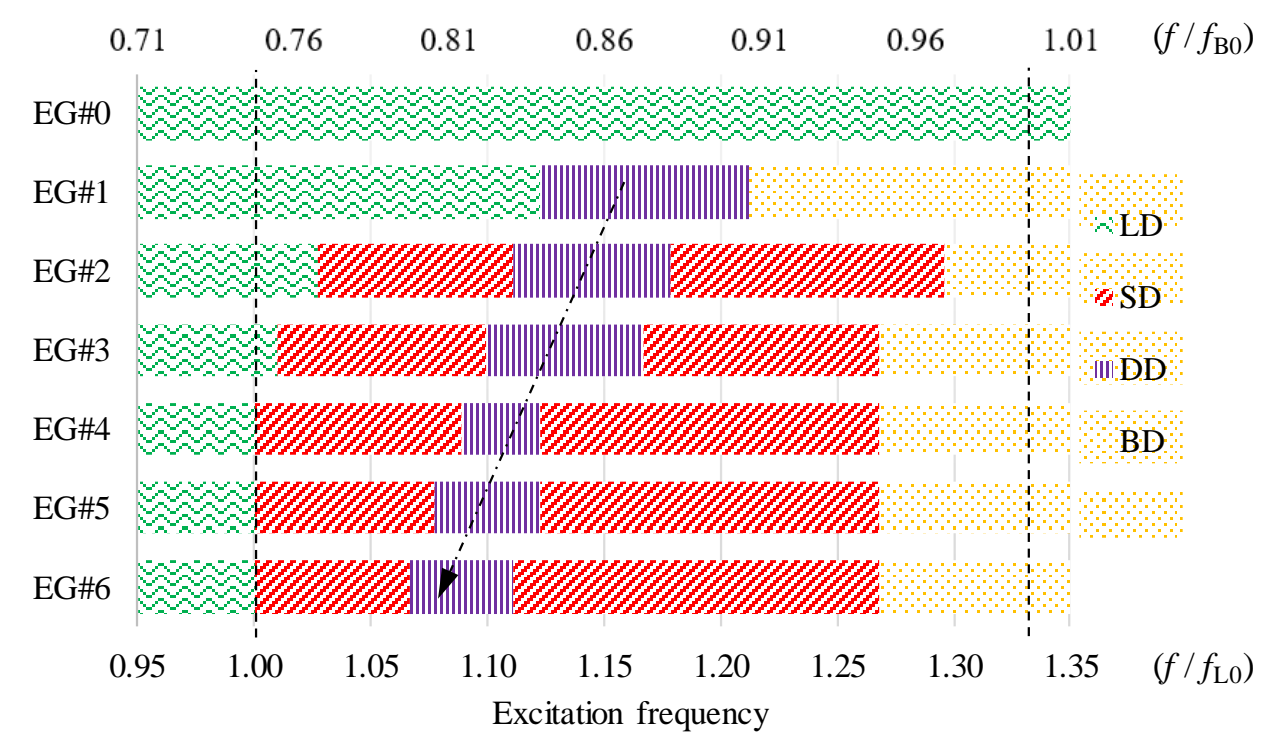

Fig. 7. Pattern variation of steady-state sloshing waves with excitation frequency in seven combinations of roll and pitch angles (LD: green; SD: red; DD: purple; PD: yellow)

In a certain excitation group, the sloshing wave pattern is very sensitive to the excitation frequency. A small change of the frequency can result in significant changes in the waveform. This, combined with the breaking waves and droplets associated with the violent sloshing, causes the coexistence of more than one wave pattern or the transition from one pattern to another. In this study, the wave pattern is classified as the waveform that dominates the wave motions.

The 1-degree-of-freedom roll excitation in EG\#0 induces only LD waves that behave almost in 2D. In EG\#1 where the pitch exists but its amplitude is small, the LD, DD and BD waves are observed. In the other excitation groups where both roll and pitch are significant, all the four wave patterns occur. This means that the interaction of sloshing waves along length and breadth directions plays an important role in generating complicated waves. Since this study focuses on 3D sloshing, only the results in EG\#1 -6 are discussed in detail.

The excitations of frequency smaller than or equal to $f_{\mathrm{L} 0}$ induce LD sloshing waves while those of frequency larger than or equal to $f_{\mathrm{B} 0}$ produce $\mathrm{BD}$ waves in all the studied cases. Under the excitations whose frequencies locate between the two resonant frequencies, significant sloshing waves are generated in both directions, and a frequency closer to the resonance of one direction induces larger waves in that direction. To explain the rationality of these phenomena, the sloshing cases under rollonly and pitch-only excitations were tested. For illustration, the peak value of $p_{2}$ (the way to get the peak pressure will be elaborated in Section 3.3) against excitation frequency is presented in Fig. 8. The two curves support the above discussions well. Note that for the pitch-only case, the maximum peak pressure does not occur at $f=f_{\mathrm{B} 0}$. This is because the actual resonant frequency of the sloshing system in the breadth direction is different from the estimated value that is based on the linear wave theory. Given the irregular shape of the tank and the high nonlinearity of the violent sloshing, this difference is 
reasonable. The way the rotation amplitude influences the sloshing is more intuitive, i.e. a larger amplitude inducing larger sloshing waves. The influences of excitation frequency and amplitude on sloshing waves are summarized in Fig. 9. In the shaded area where the excitation frequency locates between $f_{\mathrm{L} 0}$ and $f_{\mathrm{B} 0}$, significant waves can be generated in both the length and breadth directions. The interaction of waves in the two directions leads to complicated 3D waves such as the DD and SD waves.

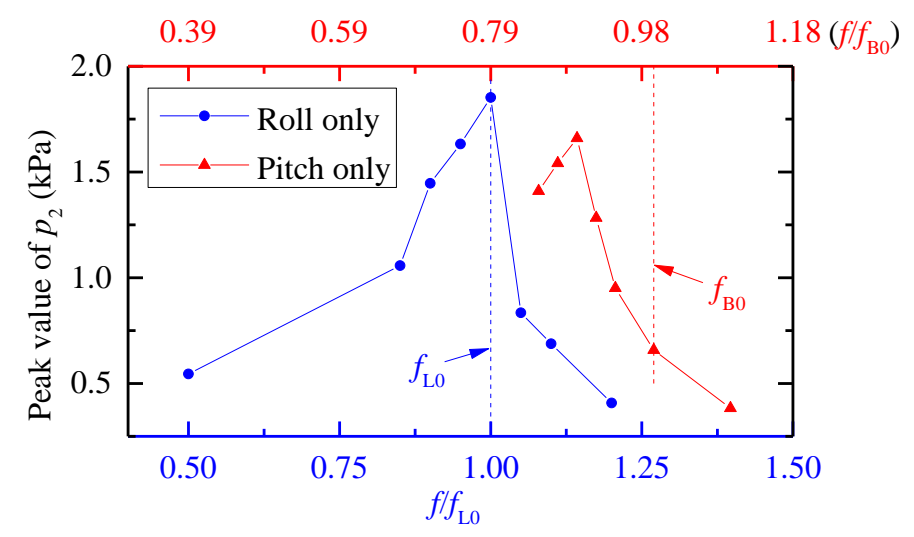

Fig. 8. Variation of sloshing pressure $p_{2}$ with excitation frequency under roll-only and pitch-only

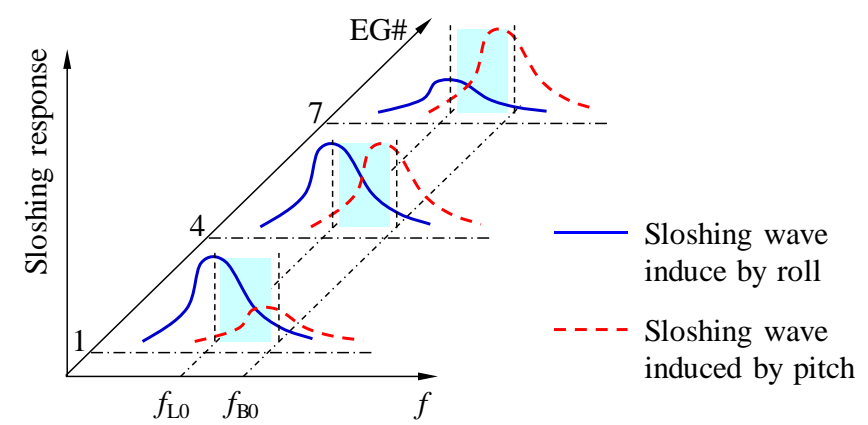

Fig. 9. Schematic view of how excitation frequency and amplitude influence sloshing responses

There is a narrow frequency band, in which the sloshing behaves as the DD wave (indicated by the purple colour in Fig. 7). For illustration, the snapshots of the case $f=1.145 f_{\mathrm{L} 0}\left(1.020 \mathrm{~Hz}, 0.858 f_{\mathrm{B} 0}\right)$ in EG\#3 are presented in Fig. 10. At $t=8.20 \mathrm{~s}$, the wave crest occurs at the right-back corner of the tank, while the trough occurs at its diagonal corner. In the next half-cycle at $t=8.60 \mathrm{~s}$, the crest and trough swap the locations. A half-cycle later $(t=9.16 \mathrm{~s})$, the wave crest occurs at the right-back corner again. This shows the diagonal movement of the sloshing wave. The frequency band at which the DD wave occurs shifts towards $f_{\mathrm{L} 0}$ from EG \#1 to \#6. This is because, with the decrease of the roll amplitude, an excitation frequency closer to $f_{\mathrm{L} 0}$ is needed to produce a sufficiently large wave in the length direction to form the diagonal wave. 


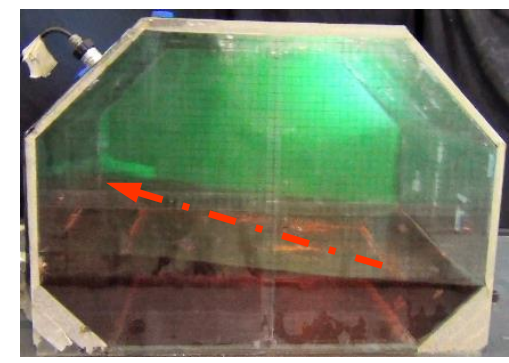

$t=0.64 \mathrm{~s}($ cycle 1$)$

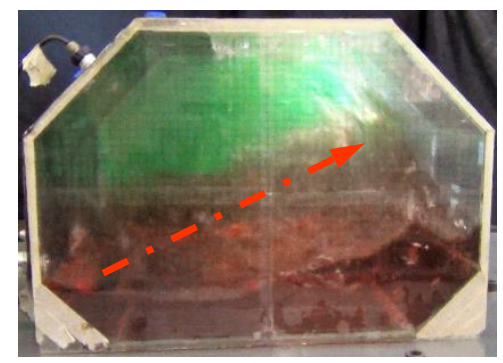

$t=8.20 \mathrm{~s}$ (cycle 8$)$

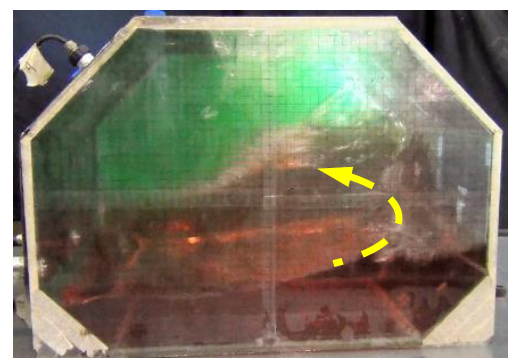

$t=2.36 \mathrm{~s}($ cycle 3$)$

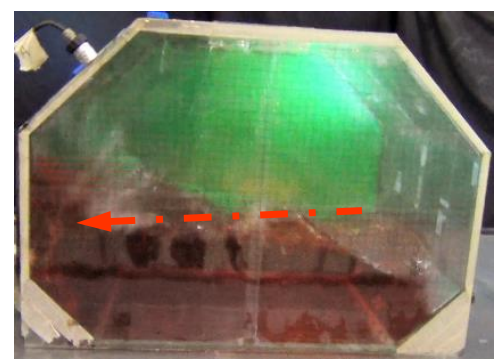

$$
t=8.60 \mathrm{~s}(\text { cycle } 9)
$$

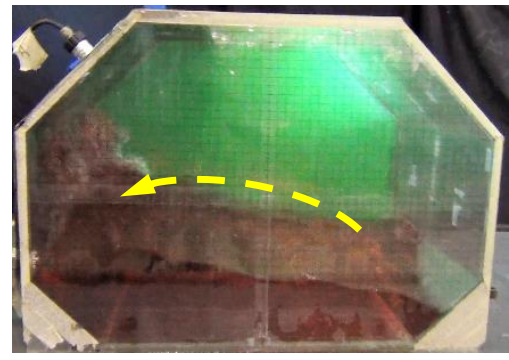

$t=2.60 \mathrm{~s}($ cycle 3$)$

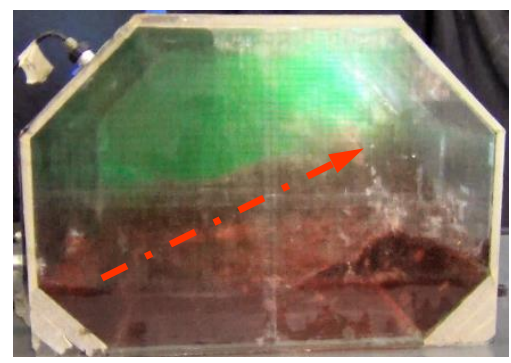

$t=9.16 \mathrm{~s}$ (cycle 9$)$

Fig. 10. Wave snapshots at typical time instants in the case $f=1.145 f_{\mathrm{L} 0}\left(1.020 \mathrm{~Hz}, 0.858 f_{\mathrm{B} 0}\right)$ in EG\#3: the top row shows the DD and SD waves at the transient phase; the bottom row shows the DD wave at the steady-state phase (the red dash-dot lines show the propagation direction of the diagonal wave with the arrows indicating the wave crest locations; the yellow dash lines show the propagation direction of the swirling wave with the arrows indicating the wave crest locations)

At the low-frequency side of the DD wave regime, the SD wave is observed in EG\#2 to \#6. For illustration, the wave snapshots at typical time instants for the case $f=1.055 f_{\mathrm{L} 0}\left(0.94 \mathrm{~Hz}, 0.791 f_{\mathrm{B} 0}\right)$ in EG\#3 are presented in Fig. 11. The wave crest locates at the right-front corner of the tank at $t=25.76 \mathrm{~s}$ and then moves to the left-back and left-front corners subsequently at $t=26.04 \mathrm{~s}$ and $26.12 \mathrm{~s}$. This shows an anticlockwise (see from the top) swirling. The swirling wave is also demonstrated by the early occurrence of the sloshing pressure at $p_{1}$ (pressure in the middle of the sidewall) than that at $p_{2}$ (see Fig. 14a). In EG\#1, the SD wave is not observed, and the sloshing transits from the DD wave to the LD wave directly. The absence of the SD wave is because, with a small pitch amplitude and an excitation frequency far from $f_{\mathrm{B} 0}$, the sloshing wave in the breadth direction is too small to interact with the wave in the length direction to form the SD wave. At the high-frequency side of the DD wave regime, the SD wave was also observed in EG\#2 to \#6, but not in EG\#1 because the pitch amplitude is small.

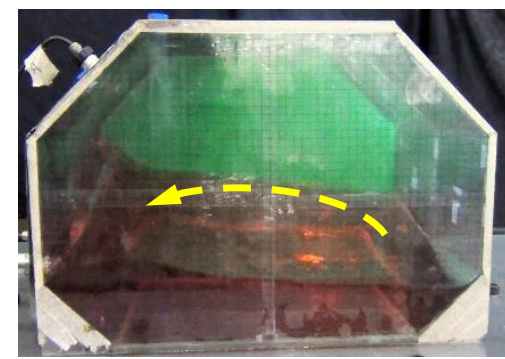

$t=1.60 \mathrm{~s}($ cycle 2$)$

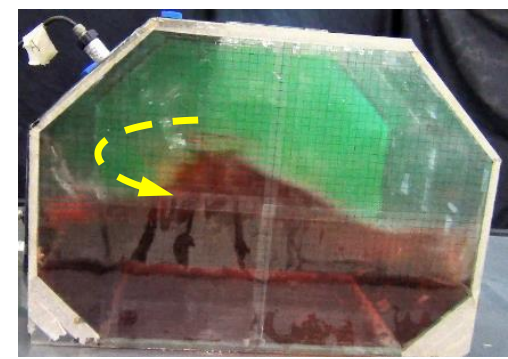

$t=2.00 \mathrm{~s}($ cycle 2$)$

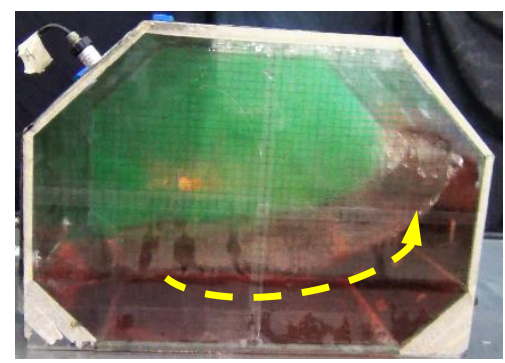

$t=2.20 \mathrm{~s}($ cycle 2$)$ 


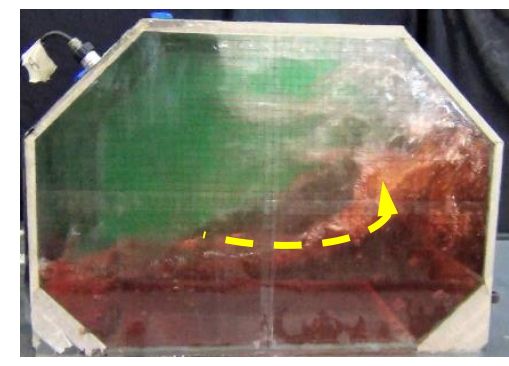

$t=25.76 \mathrm{~s}$

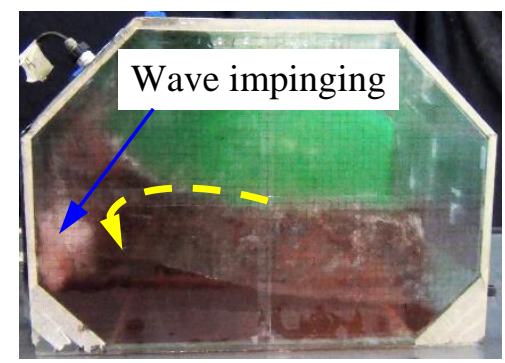

$t=26.12 \mathrm{~s}$

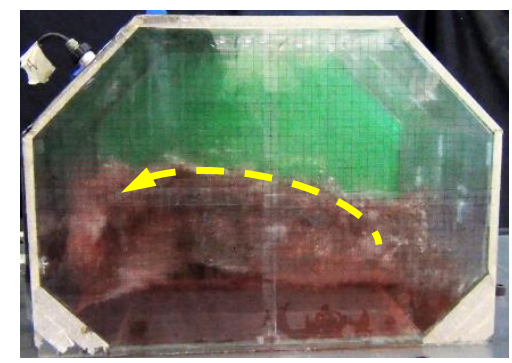

$t=26.04 \mathrm{~s}$

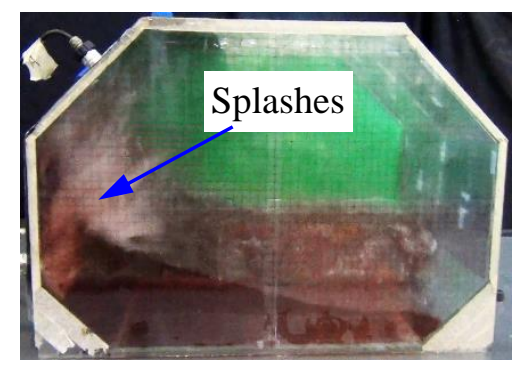

$t=26.16 \mathrm{~s}$

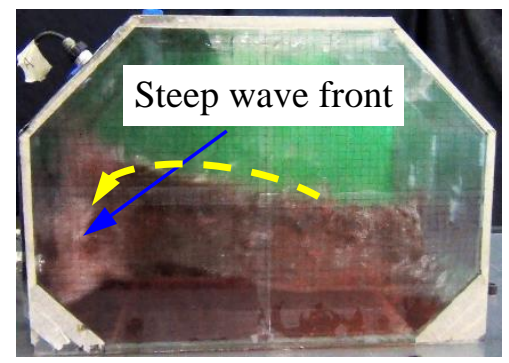

$t=26.08 \mathrm{~s}$

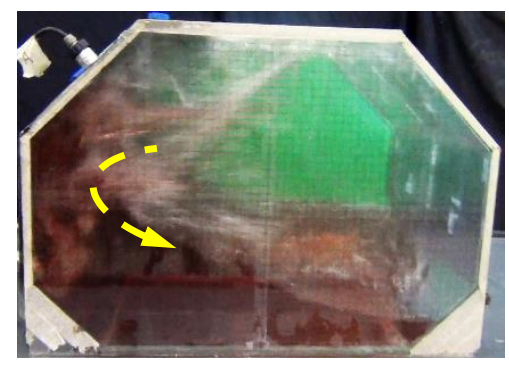

$t=26.24 \mathrm{~s}$

Fig. 11. Wave snapshots at typical time instants in the case $f=1.055 f_{\mathrm{L} 0}\left(0.94 \mathrm{~Hz}, 0.791 f_{\mathrm{B} 0}\right)$ in EG\#3: the first row shows the swirling wave at the transient phase; the second and third rows show the swirling wave at the steady-state phase (the yellow dash lines show the propagation direction of the swirling wave with the arrows indicating the wave crest locations)

All the SD wave cases have two things in common: 1) both the roll and pitch amplitudes are significant (the ratio of pitch amplitude to roll amplitude ranges from 0.5 to 2 for the studies cases); 2 ) the excitation frequency locates between $f_{\mathrm{L} 0}$ and $f_{\mathrm{B} 0}$. These two conditions give rise to significant sloshing waves in the length and breadth directions (demonstrated in Fig. 9), the superposition of which produces the SD wave. The sloshing experiments under translational excitations (Luo et al., 2016) also produced significant waves in two directions but did not observe the swirling wave. It is hypothesized that the rotational motion of the tank imposes necessary initial conditions of the sloshing fluid to trigger the swirling wave. The swirling wave can be a concern because it is always accompanied with wave impingements. As shown in the bottom row of Fig. 11, in the propagation of the swirling wave a steep wavefront is formed and about to break at $t=26.08 \mathrm{~s}$. It then impinges on the front wall near the tank corner $(t=26.12 \mathrm{~s})$ and produces wave splashes $(t=26.16 \mathrm{~s})$. The interaction of wave swirling with tank walls is sketched in Fig. 3c. The wave impingement induces violent impact pressures near the tank corner, which can cause serious damages to the interior layer of the membrane tank.

\subsubsection{Wave patterns in the transient phase}

The sloshing pattern in the transient phase is generally more complicated. The sloshing waves in the first $1-2$ cycles tend to follow the tank motion. Specifically, the incipient sloshing wave in EG\#1 cases is the LD wave because the primary tank motion is roll, and those in EG\#4 and EG\#6 are DD and BD waves, respectively. After that, the sloshing wave may keep the incipient pattern and develop its 
311 amplitude until the steady-state amplitude. This phenomenon is observed in the LD wave regime in

312 EG\#1 to \#3 and the BD wave regime in EG\#5 and \#6. In other cases, the sloshing wave in the transient

313 phase changes its form and evolves into the steady-state wave pattern. For example, in the case $f=1.145$

$314 f_{\mathrm{L} 0}\left(0.858 f_{\mathrm{B} 0}\right)$ in EG\#3 (see the wave snapshots in the top row of Fig. 10), the rotational excitation

315 induces the nearly-diagonal wave in the first cycle, which then evolves to the swirling wave in the

316 subsequent transit cycles and the DD wave in the steady-state cycles. It is noteworthy that the swirling

317 wave occurs frequently in the transient phase, which causes large pressure peaks (see Fig. 6).

\section{3.3. Sloshing pressure}

319 The sloshing pressures that are of great concern in the practical design are examined in this section.

320 In data processing, the peak pressure of each sloshing cycle was first extracted. The maximum of the

321 peaks in the transient-phase cycles and the mean of the highest one-third of the pressure peaks (Fillon

322 et al., 2011) in the steady-state phase are computed as the indicators of the peak pressures for a particular

323 sloshing case. In the BD wave pattern, the propagation direction of the sloshing wave is nearly parallel

324 to the measuring diaphragms of the pressure sensors (wave attack angle close to 90 degrees). This leads

325 to that relatively small pressures were recorded on the sensors although the sloshing waves in some

326 cases are moderate or violent. Hence, the pressures of the BD sloshing waves are shown for

327 completeness of the presentation, but not discussed in detail. Based on the pressure results in the other

328 wave patterns, the characteristics of sloshing pressures are discussed.

\subsubsection{Variation of peak pressure with excitation amplitude and frequency}

330 The statistically-averaged peak values of the steady-state pressures at $p_{1}$ and $p_{2}$ are presented in Fig.

331 12. When the excitation frequency approaches $f_{\mathrm{L} 0}$ from the low-frequency side, the pressure peaks at 332 the tank corner, i.e. $p_{2}$, increase in all the excitation groups. After $f_{\mathrm{L} 0}, p_{2}$ in EG\#1 goes down, while it

333 continues to increase in the other excitation groups. The main reason that causes this difference is the

334 swirling wave. In EG\#1, after the excitation frequency exceeds $f_{\mathrm{L} 0}$, the sloshing waves induced by roll

335 become smaller. Although the excitation frequency gets closer to $f_{\mathrm{B} 0}$, the waves excited by pitch are not

336 large because of the small pitch amplitude. This leads to that the intensity of the resultant sloshing wave reduces monotonically with the increase of the excitation frequency after $f_{\mathrm{L} 0}$. In EG\#2 to \#6, the sloshing wave evolves into the SD wave after the excitation frequency exceeds $f_{\mathrm{L} 0}$. The $\mathrm{SD}$ wave causes violent impingements and hence large impact pressures at the tank corner. Indeed, the SD wave regime records the maximum peak, which is around $3 \mathrm{kPa}$ in EG\#2 to \#6. The sloshing wave then turns into the DD wave that moves along the diagonal direction of the tank (see the bottom panel of Fig. 10). The DD wave is generally much less energetic than the SD wave and hence causes much smaller sloshing pressures in all the excitation groups.

With a further increase of the excitation frequency, the sloshing wave goes into the high-frequency SD wave regime. The pressure peaks increase markedly to a level comparable to that in the low- 
frequency SD regime, making the $p-f$ curve to be an " $M$ " shape, for which the two spires happen at the two SD regimes. This manifests the potential destructiveness of the swirling wave. It has been demonstrated in Arai et al. (2018) that swirling waves can happen in membrane tanks in real seaways. Therefore, the design for the interior layers of membrane tanks against sloshing impacts should consider the swirling wave, because this particular waveform can cause severer impact pressures compared to other types of sloshing waves. Further increasing the excitation leads to the BD waves. By observing the experimental videos (not presented), BD waves are in general less violent than the LD and SD waves.

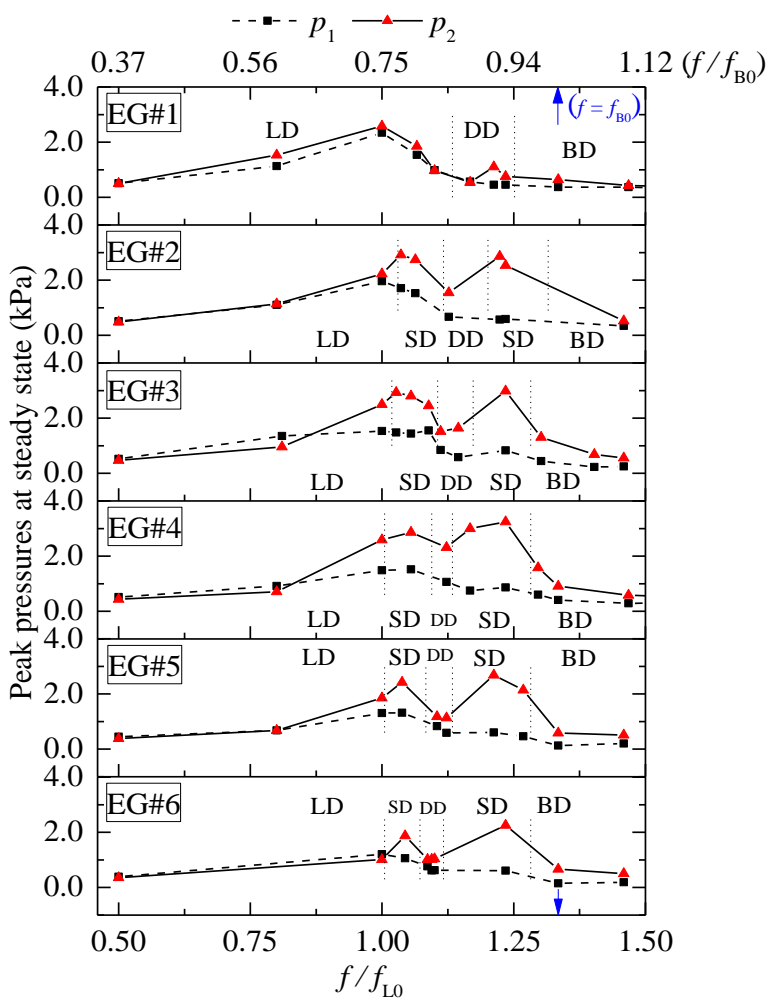

Fig. 12. Comparison of the peak values of the steady-state pressures at $p_{1}$ and $p_{2}$

For the steady-state pressures in the middle of the tank wall $\left(p_{1}\right)$, the maximum peak occurs at an excitation frequency near $f_{\mathrm{L} 0}$. In EG\#2 to \#6, the " $\mathrm{M}$ " shape of the $p-f$ curve is also visible although the second spire of the " $\mathrm{M}$ " is not high. For a particular case that produces violent sloshing, $p_{1}$ is normally much smaller than $p_{2}$, with the ratio ranging from $20 \%$ to $50 \%$. The exception is EG\#1, where the LD wave causes similar impact pressures in the middle of the side wall and at the tank corner. As can be seen from Fig. 12, the cases where $p_{1}$ and $p_{2}$ show significant differences locate mainly in the SD wave regimes. This is because the swirling wave impinges the tank wall ahead of the wavefront (e.g. the front wall sketched in Fig. 3c), inducing a large impact pressure near the tank corner. In contrast, the impact in the middle of the side wall $\left(p_{1}\right)$ is induced by the skim-over flow and hence has a much smaller peak.

In most of the violent sloshing cases, the wave pattern at the transient phase is different from that in the steady-state phase. To assess how sloshing pressures at the transient phase behave, the peak values of $p_{2}$ (the more critical one) at the transient phase are compared with the peaks at the steady-state phase 
in Fig. 13. The discussions are focused on the cases that record violent impact pressures, i.e. the cases with excitation frequency ranging from $f_{\mathrm{L} 0}$ to $f_{\mathrm{B} 0}$. In $\mathrm{EG} \# 1$, the transient and steady-state peak pressures are close. This is because the sloshing waves in the transient phase move in the same pattern as the steady-state waves, and they just develop the wave amplitude and reach the intensity comparable to that of the steady state. In the other excitation groups, the transient and steady-state pressures show evident differences. Specifically, the transient peak pressure is smaller than the respective steady-state value in the SD wave regime (see Fig. 5). This is because the swirling waves in the steady state are always very violent and hence induce larger pressures than the transient cycles irrespective of the waveform. In the DD wave regime, however, the peak pressure is observed to be larger than that in steady state because of the occurrence of the swirling wave in the transient phase (see Fig. 6).

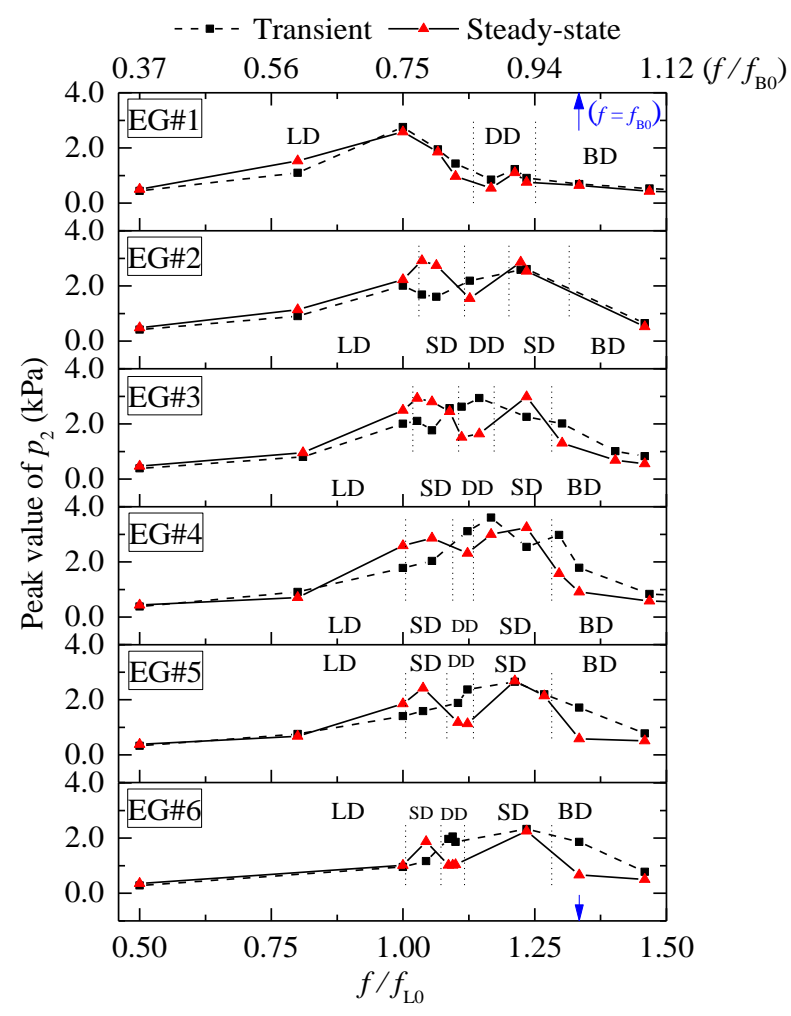

Fig. 13. Comparison of the peak values of the steady-state and transient pressures at $p_{2}$

\subsubsection{Features of pressure signals and the implication}

The peak pressures in different wave patterns are discussed in the previous section. The features of the pressure signals for swirling and diagonal waves are analysed here. For illustration, the pressure results of one sloshing cycle in the cases of $f=1.055 f_{\mathrm{L} 0}\left(0.791 f_{\mathrm{B} 0}\right), 1.145 f_{\mathrm{L} 0}\left(0.858 f_{\mathrm{B} 0}\right)$ and $1.235 f_{\mathrm{L} 0}$ $\left(0.925 f_{\mathrm{B} 0}\right)$ in EG\#3 are presented in Fig. 14. These cases correspond to the low-frequency SD wave, DD wave and high-frequency SD wave, respectively. The impulse-like impact that is characterized by a short rise time and a large peak is recorded in the two SD wave cases (see the top and bottom panel of Fig. 14). From the bottom row of Fig. 11, it can be seen that a steep wave front is formed and about to break just before the wave impact occurs. This scenario is similar to Case $\mathrm{C}$ sloshing in Delorme et 
al. (2009). The pressure signals in these two SD wave cases show fluctuations, which are attributed to the aerated flow (see the "white" wave in the snapshots at $t=26.08 \mathrm{~s}$ and $26.12 \mathrm{~s}$ in Fig. 11). In contrast, the pressure signal in the DD wave case (the middle panel of Fig. 14) exhibits a larger rise time and a much smaller peak. This is because a DD wave, during the impact process, climbs up/down along the tank corner and applies moderate loads. The rise time of the pressure signal is the key parameter for describing dynamic amplification of structural responses (DNV, 2016), and hence should be considered carefully in the design by taking the structural properties into account.
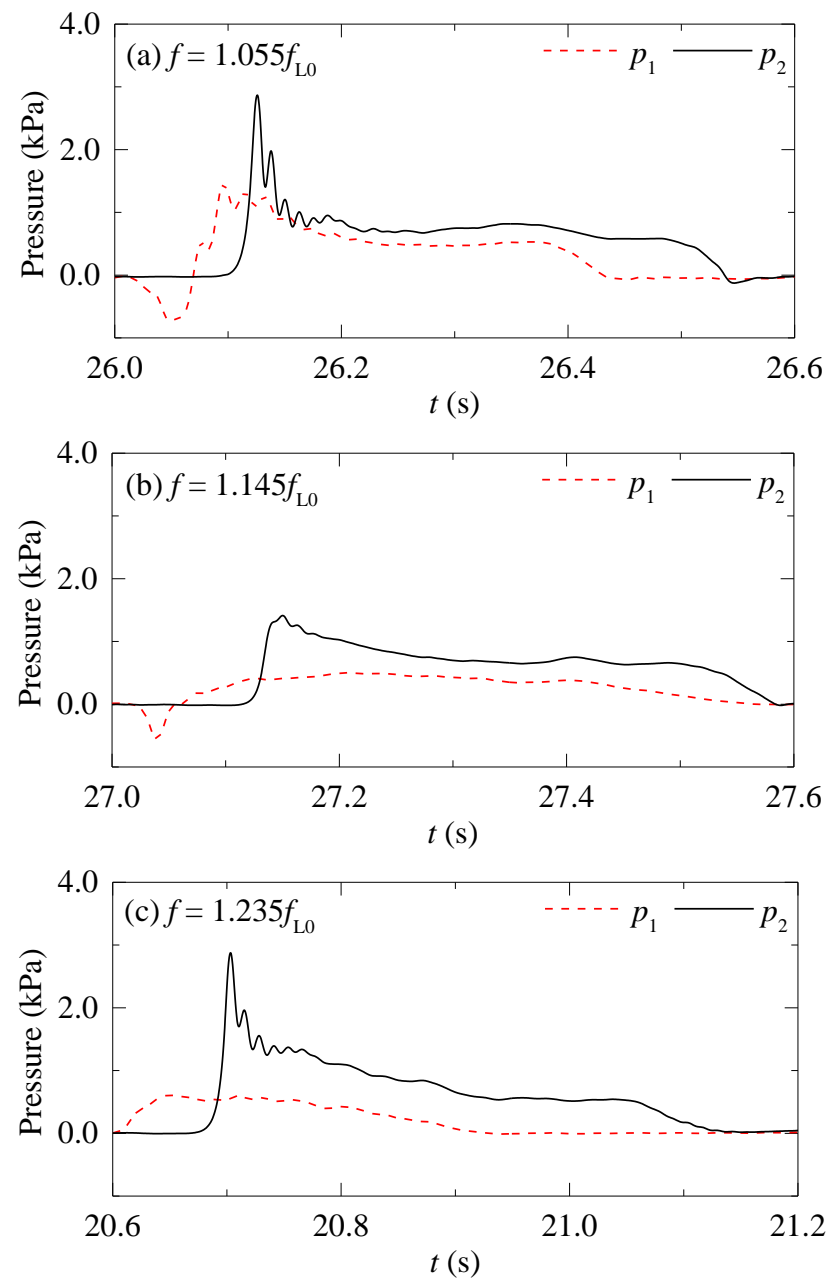

\section{Conclusions}

This study investigates the violent sloshing in a scaled membrane-type LNG tank whose length-tobreadth ratio is around 1.4. The coupled roll and pitch motions, which have been rarely documented in the literature, are considered. Seven groups of roll and pitch amplitudes are tested. In each group, 9 11 excitation frequencies are studied with refined frequency points near the transition of the waveform. In each case, the pitch and roll have the same frequency. The sloshing wave patterns and impact pressures are investigated in detail. The key findings are summarized below: 
- The sloshing waves in the studied cases exhibit four distinct patterns, i.e. the length-dominant wave, swirling wave, diagonal-dominant wave, and breadth-dominant wave. The occurrence of a particular pattern is related to the pitch and roll amplitudes and excitation frequency.

- Based on the studies cases, the swirling wave is excited when two conditions are satisfied: (1) both the roll and pitch amplitudes are significant (the ratio of pitch amplitude to roll amplitude within $[0.5,2]$ for the studies cases); (2) the excitation frequency locates between $f_{\mathrm{L} 0}$ and $f_{\mathrm{B} 0}$. These two conditions ensure significant sloshing waves in the length and breadth directions, which provide the necessary condition for the generation of a swirling wave. Since the swirling wave was not observed in the sloshing experiments under 2-degree-of-freedom translational excitations (Luo et al., 2016), it is hypothesized that the rotational motion of the tank imposes necessary initial conditions to trigger the swirling wave.

- The swirling wave impinges on the walls near the tank corner, inducing intensive impact pressures. Based on the cases considered in this study that used two pressure sensors, the sloshing pressure at the tank corner is $2-5$ times larger than that in the middle of the wall. The implication is that the corner of a membrane-type LNG tank should be enforced in practical design. A future work direction is to use more pressure sensors to measure and quantify the spatial distribution of sloshing pressures near the tank corner.

- The waveforms in the transient phase are generally more complicated, being closely related to the direction of tank moving and the steady-state wave pattern. The peak pressures of the transient and steady-state phases in a particular case show evident differences in the cases recording violent 3D sloshing. For a particular excitation group, the maximum of the peaks of transient pressures for different excitation frequencies is very close to that of steady-state pressures.

\section{Acknowledgement}

The experimental work was financially supported by the funding and technical support of the Maritime and Port Authority of Singapore (Grant WBS No. R-302-000-012-490), the National University of Singapore and the American Bureau of Shipping. The first author appreciates the Belt and Road Special Foundation of the State Key Laboratory of Hydrology-Water Resources and Hydraulic Engineering (Project No: 2019491711) and the Open Research Funding SKHL1710 and SKHL1712 from the State Key Laboratory of Hydraulics and Mountain River Engineering in Sichuan University, and thanks to Dr Jun Lin Too and Mr Perry Teo in helping to conduct the experiments.

\section{References}

Arai, M., Yoshida, T., Ando, H., 2018. Experimental and numerical study of sloshing and swirling behaviors in partially loaded membrane LNG tanks, The 13th International Marine Design Conference (IMDC 2018). CRC Press, Helsinki, Finland. 
Bai, W., Liu, X., Koh, C.G., 2015. Numerical study of violent LNG sloshing induced by realistic ship motions using level set method. Ocean Engineering 97, 100-113.

443 Bulian, G., Botia-Vera, E., Souto-Iglesias, A., 2014. Experimental sloshing pressure impacts in ensemble domain: Transient and stationary statistical characteristics. Physics of Fluids 26 (3), 032102.

Chen, B.-F., Wu, C.-H., 2011. Effects of excitation angle and coupled heave-surge-sway motion on fluid sloshing in a three-dimensional tank. Journal of Marine Science and Technology 16 (1), 22-50.

Chen, Z., Zong, Z., Li, H.T., Li, J., 2013. An investigation into the pressure on solid walls in 2D sloshing using SPH method. Ocean Engineering 59, 129-141.

Delorme, L., Colagrossi, A., Souto-Iglesias, A., Zamora-Rodríguez, R., Botía-Vera, E., 2009. A set of canonical problems in sloshing, Part I: Pressure field in forced roll-comparison between experimental results and SPH. Ocean Engineering 36 (2), 168-178.

DNV, 2016. Sloshing analysis of LNG membrane tanks.

Faitinsen, O.M., 1978. A numerical nonlinear method of sloshing in tanks with two-dimensional flow. Journal of Ship Research 22 (03), 193-202.

Faltinsen, O.M., Rognebakke, O.F., Timokha, A.N., 2005. Classification of three-dimensional 103.

Fillon, B., Diebold, L., Henry, J., Derbanne, Q., Baudin, E., Parmentier, G., 2011. Statistical postprocessing of long-duration sloshing test, The Twenty-first International Offshore and Polar Engineering Conference. International Society of Offshore and Polar Engineers.

Jin, X., Lin, P., 2019. Viscous effects on liquid sloshing under external excitations. Ocean Engineering 171, 695-707.

Koh, C.G., Gao, M., Luo, C., 2012. A new particle method for simulation of incompressible free surface flow problems. International Journal for Numerical Methods in Engineering 89 (12), 1582-1604.

Lee, D.H., Kim, M.H., Kwon, S.H., Kim, J.W., Lee, Y.B., 2007. A parametric sensitivity study on LNG tank sloshing loads by numerical simulations. Ocean Engineering 34 (1), 3-9.

Liu, D., Lin, P., 2008. A numerical study of three-dimensional liquid sloshing in tanks. Journal of Computational Physics 227 (8), 3921-3939. interactions. Science China Physics, Mechanics \& Astronomy 62 (8). Coupling Problems. International Journal of Offshore and Polar Engineering (IJOPE) 26 (1), 26-32.

Lloyd'sRegister, 2008. Guidance on the operation of membrane LNG ships to reduce the risk of damage due to sloshing. Lloyd's Register, London. amplitude in a rectangular sloshing tank. Ocean Engineering 156, 500-511. 
Luo, M., Koh, C.G., Bai, W., 2016. A three-dimensional particle method for violent sloshing under regular and irregular excitations. Ocean Engineering 120, 52-63.

Malenica, S., Diebold, L., Kwon, S.H., Cho, D.-S., 2017. Sloshing assessment of the LNG floating units with membrane type containment system where we are? Marine Structures 56, 99-116.

Meng, Z.-F., Wang, P.-P., Zhang, A.M., Ming, F.-R., Sun, P.-N., 2020. A multiphase SPH model based on Roe's approximate Riemann solver for hydraulic flows with complex interface. Computer Methods in Applied Mechanics and Engineering 365, 112999.

Mokhatab, S., Mak, J.Y., Valappil, J.V., Wood, D.A., 2013. Handbook of liquefied natural gas. Gulf Professional Publishing.

Pal, P., Bhattacharyya, S.K., 2010. Sloshing in partially filled liquid containers-Numerical and experimental study for 2-D problems. Journal of Sound and Vibration 329 (21), 4466-4485.

Souto-Iglesias, A., Botia-Vera, E., Bulian, G., 2012. Repeatability and two-dimensionality of model scale sloshing impacts, The Twenty-second International Offshore and Polar Engineering Conference. International Society of Offshore and Polar Engineers.

Souto-Iglesias, A., Bulian, G., Botia-Vera, E., 2015. A set of canonical problems in sloshing. Part 2: Influence of tank width on impact pressure statistics in regular forced angular motion. Ocean Engineering 105, 136-159.

Wang, K., 2010. Loss prevention through risk assessment surveys of LNG carriers in operation, under construction, conversion and repair. BMT Msrine \& Offshore Surveys, Tokyo, Japan.

Wu, C.-H., Chen, B.-F., 2009. Sloshing waves and resonance modes of fluid in a 3D tank by a timeindependent finite difference method. Ocean Engineering 36 (6-7), 500-510.

Wu, C.-H., Chen, B.-F., Hung, T.-K., 2013. Hydrodynamic forces induced by transient sloshing in a $3 \mathrm{D}$ rectangular tank due to oblique horizontal excitation. Computers \& Mathematics with Applications 65 (8), 1163-1186.

Xue, M.-A., Zheng, J., Lin, P., Yuan, X., 2017. Experimental study on vertical baffles of different configurations in suppressing sloshing pressure. Ocean Engineering 136, 178-189.

Xue, M.A., Lin, P., 2011. Numerical study of ring baffle effects on reducing violent liquid sloshing. Computers \& Fluids 52, 116-129.

Zhao, D., Hu, Z., Chen, G., Lim, S., Wang, S., 2018. Nonlinear sloshing in rectangular tanks under forced excitation. International Journal of Naval Architecture and Ocean Engineering 10 (5), 545-565.

Zheng, X., Shao, S., Khayyer, A., Duan, W., Ma, Q., Liao, K., 2017. Corrected First-Order Derivative ISPH in Water Wave Simulations. Coastal Engineering Journal 59 (1), 1750010-1750011-17500101750029. 